\title{
Myringoplasty with Morphological Rationale of Application of Xenoperitoneum Decellularized Matrix in Experiment
}

\author{
Dias Yesniyazov, Tussupbekova Maida, Nurkasi Abatov, Yekaterina Yukhnevich*, Ruslan Badyrov \\ Department of Surgical Diseases, Karaganda Medical University, Karaganda, Kazakhstan
}

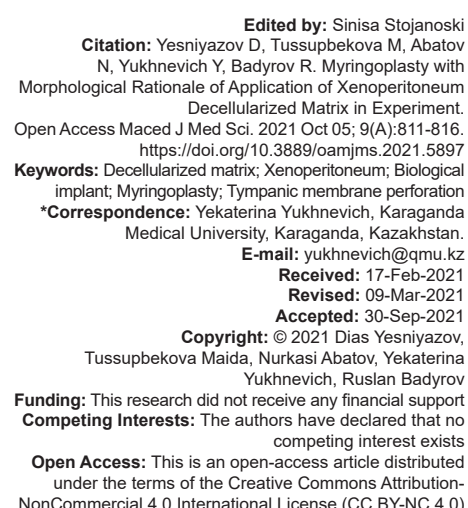

Edited by: Sinisa Stojanosk Edited by: Sinisa Stojanosk
Citation: Yesniyazov D, Tussupbekova M, Abatov Citation: Yesniyazov D, Tussupbekova M, Abatov
N, Yukhnevich Y, Badyrov R. Myringoplasty with Morphological Rationale of Application of Xenoperitoneum Morphological Rationale of Application of Xenoperitoneum
Decellularized Matrix in Experiment. Open Access Maced J Med Sci. 2021 Oct 05; 9(A):811-816
https://doi.org/10.3889/oamjms.2021.5897 Keywords: Decellularized matrix; Xenoperitoneum; Biological implant; Myringoplasty; Tympanic membrane perforation *Correspondence: Yekaterina Yukhnevich, Karaganda Medical University, Karaganda, Kazakhstan E-mail: yukhnevich@qmu.kz Received: 17-Feb-202 Accepted: 30 -Sep-202 Copyright: ๑) 2021 Dias Yesniyazov Copyright: @ $\odot 2021$ Dias Yesniyazov,
Tussupbekova Maida, Nurkasi Abatov, Yekaterina Yukhnevich, Ruslan Badyrov Funding: This research did not receive any financial support Competing Interests: The authors have declared that no competing interest exists Open Access: This is an open-access article distributed under the terms of the Creative Commons Attribution-

\begin{abstract}
AIM: The aim of the study is to create a myringoplasty technique with decellularized matrix of xenoperitoneum in an experiment on rabbits with morphological rationale of a new biological material application.

METHODS: Sixty rabbits were included in research. The animals were randomly divided into two groups for use the bioimplant-decellularized matrix of xenoperitoneum and control. Each subgroup corresponded to the observation period and the removal of the animal from the experiment. Observation period was 7 days, 21 days, and 30 days after myringoplasty.

RESULTS: The maturation of connective tissue in both groups was completed by the $30^{\text {th }}$ day. However, the change in the exudative phase into the proliferative phase occurred faster and the dynamics of reparative processes after myringoplasty was better in experimental group.

CONCLUSION: The use of the decellularized matrix of xenoperitoneum had better reparative properties.
\end{abstract}

\section{Introduction}

There are many different biological materials that differ both in the primary material and in the processing methods - decellularization and sterilization processes [1].

Despite a sufficient number of works on the problem of xenoimplants using, the ambiguity of the data presented by different scientists should be recognized, and the lack of studies on the use of the decellularized matrix of xenoperitoneum for tympanic membrane defects repair also determines the relevance of the planned study [2], [3], [4], [5].

\section{Aim of study}

This study aims to create a myringoplasty technique with decellularized matrix of xenoperitoneum in an experiment on rabbits with morphological rationale of a new biological material application.

\section{Materials and Methods}

For the assigned tasks implementation, an experiment was carried out on 60 sexually mature rabbits of both sexes with $1500 \pm 300 \mathrm{~g}$ of weight. The animals were randomly assigned into two groups and three subgroups. The $1^{\text {st }}$ group included the animals with the used bioimplant-decellularized matrix of xenoperitoneum (the implant thickness was 500 microns); the $\mathrm{II}^{\text {nd }}$ group was the control group, where preserved dura mater was used as a plastic material. Each subgroup corresponded to the observation period and the removal of the animal from the experiment. Observation period was 7 days, 21 days, and 30 days after myringoplasty. The experiment was carried out on the left ears of the animals; therefore, the right ears served as a control for clinical and morphological manifestations comparing.

The absence of external signs of the disease and the homogeneity of groups by body weight $( \pm 10 \%)$ was considered as a criterion for acceptable randomization. Individual identification was carried out by the assigning of individual number with a dye label on the right auricle of each animal. The basic rules of maintenance and care corresponded to the standards of the Guide for Care and Use of Laboratory Animals: Eight edition. ILAR publication, 2012, National Academy Press. All routine animal care procedures were performed in accordance with standard operating procedures. 
Histological material was taken from the area of the tympanic membrane with an implant for morphological examination after the animals removing from the experiment after 7 days, 21 days, and 30 days. Experimental work with laboratory animals was carried out in accordance with the current order of the Ministry of Health and the rules for preclinical studies biomedical experiments conducting in compliance with the international principles of the European Convention for the Protection of Vertebrate Animals used for experimental and other scientific purposes.

To create an experimental model, the rabbits were chosen as the animals in which a defect of the tympanic membrane can be simulated and it can be performed the myringoplasty using the various types of implants of biological origin followed by an assessment of morphological changes in the implantation zone and an assessment of the reparative process.

Having studied the available methods of experimental operations (myringoplasty) on animals, we are faced with the fact that the existing methods cannot be implemented for the assigned tasks [3], [6]. This situation prompted us to develop our own method of myringoplasty on experimental animals (rabbits). The essence of the proposed technique consisted in performing surgery under general anesthesia with Ketamine $25 \mathrm{mg}$ intravenously. A horizontal incision $1 \mathrm{~cm}$ long was made at the anterior edge of the auricle bottom (Figure 1a).

After diluting the edges of the wound using a retractor, the edges of the wound are bluntly parted in the direction of the initial incision, until the anterior wall of the external auditory meatus is exposed. After careful hemostasis, an incision of the external auditory meatus was made in the cartilaginous part at the place of its transition to the bone one. Thereby, we obtained a good view of the bony part of the auditory meatus (Figure 1b)

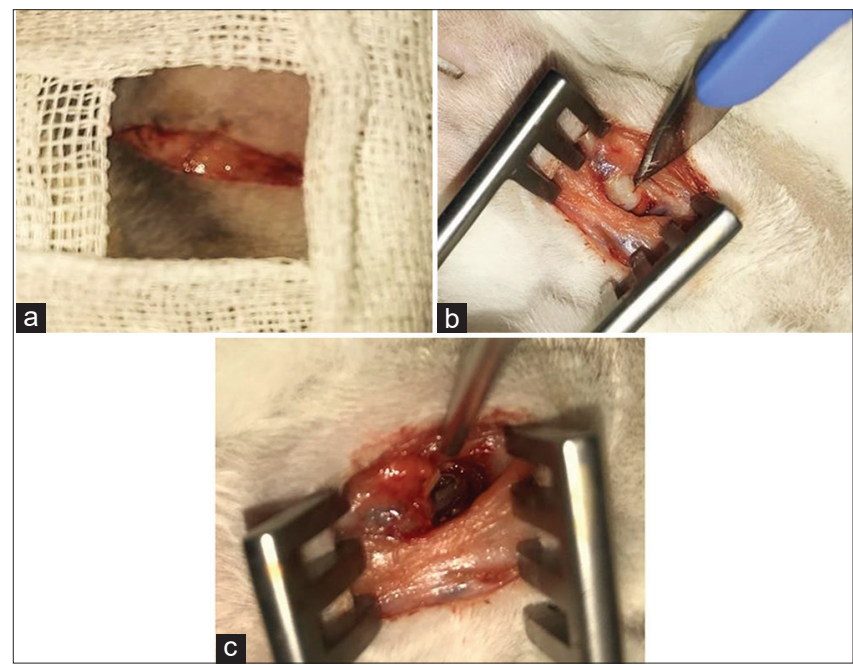

Figure 1: (a) Incision at the auricle bottom, (b) visualization of the border of the transition of the cartilaginous part of the auditory meatus into the bone, (c) removal of the outer wall of the auditory meatus
As you know, the tympanic membrane of rabbit is located at an angle of $90^{\circ}$ in relation to the entrance to the auditory meatus [7]. The outer bony wall of the auditory meatus was removed for more convenient access using bone forceps, and it manipulation provided a good view of the tympanic membrane without using the ear funnel (Figure 1c).

After careful cleaning of the external auditory meatus, the tympanic membrane was torn in the tensioned part with a needle from a tympanoplasty set. It was important to remove more than $50 \%$. It is necessary to take into account the existing statement that acute post-traumatic perforation of the tympanic membrane closes within 7-10 days independently, however, the perforations of the tympanic membrane, occupying $25 \%$ of the damaged area or more (one or more quadrants), do not close even in 30 days after injury [4]. After removing a part of the tympanic membrane, we placed the flap using the overlay method. The nature of flap was the biomaterial of decellularized matrix of xenoperitoneum with 500 microns thickness. The operation was completed by applying a silicone strip to fix the flap and Merocel sponge. Then, we match the edges of the auditory meatus, the wound edges were taken in by interrupted sutures.

\section{Results}

To assess the structural changes of the defect zone using a preserved dura mater or decellularized matrix of xenoperitoneum after myringoplasty in the experiment, a comparative analysis of the histological picture was carried out at different times of the experiment: On the $7^{\text {th }}$ day, $24^{\text {th }}$ day, and on the $30^{\text {th }}$ day after the end of the experiment. The obtained material was carried out according to the well-known classical histological technique. Paraffin sections with a thickness of 5-6 microns were made and stained with hematoxylin and eosin. The material was studied on a computerized microscope with digital microphotography of histological preparations "Leica Microsystems" at a magnification of 100, 200, and 400 times.

The morphological study was carried out by a simple blind method; each group of animals was numbered according to the terms of the experiment. After the histological description, the groups of the experiment were deciphered and a comparative analysis of the morphological picture was carried out when using the preserved dura mater or decellularized xenomedial matrix in the area of the tympanic membrane defect.

After the end of the experiment on the $7^{\text {th }}$ day, the formation of soft connective tissue with loosely intertwining fibers was noted in the group after myringoplasty with the use of preserved dura mater 
in the zone of contact with the walls of the tympanic membrane tissue defect (Figure 2a). It should be noted that infiltration by macrophages and lymphocytes was observed in the marginal zones in the implantation area (Figure 2b). Young granulation tissue was formed with ingrowth into the structure of the underlying tissue of the tympanic membrane in the main part of the implantation zone, along the entire surface of fixation of the preserved dura mater (Figure $2 c$ and $d$ ).

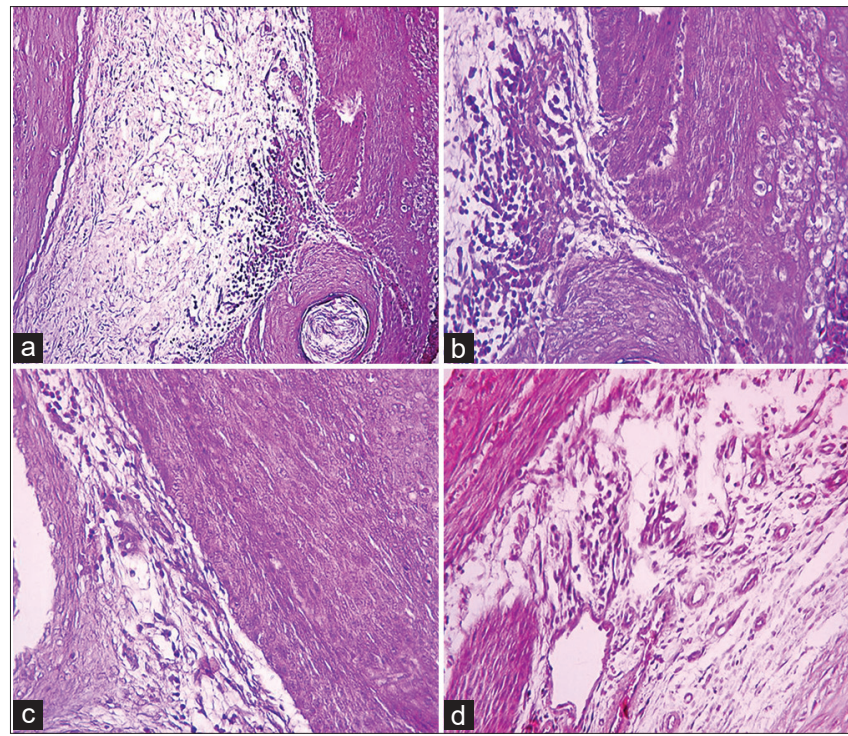

Figure 2: Tympanic membrane (experiment, Group 4.1; 5.1) - zone of plastic manipulation using preserved dura mater, the $7^{\text {th }}$ day: (a-d) The defect zone is represented by delicate intertwining fibers of connective tissue; (a-c) the edge zone of the implant with focal infiltration by lymphocytes; (d) the formation of vascularized granulation tissue. Staining: Hematoxylin and eosin. Magnification: $a-\times 100 ; b, c-\times 200 ; d-400$

In other series of experiments in this group, the maturation of granulation tissue was also well traced throughout the entire zone of the tympanic membrane defect when using the preserved dura mater as an implant on day 7. Microscopically, structural changes were represented by the formation of loose connective tissue with intertwining fibers containing current walled vessels of the capillary type; diffuse lymphoid infiltration along the marginal zone of the implant remained (Figure 3). These morphological changes can be assessed as a reactive compensatory-adaptive process in implantation zone of the preserved dura mater.

Histological study of the tympanic membrane material on the $21^{\text {st }}$ day (experiment, group 01.1.1) after myringoplasty of the defect zone using the tissue of preserved dura mater revealed maturation and formation of delicate fibrous connective tissue in the stroma with moderate infiltration by lymphocytes and macrophages, the zone vascularization by thin- walled vessels with diffuse lymphoid infiltration was well traced (Figure 3). Diffuse lymph-leukocyte infiltration registered in the border zone of the implant and the tissue of the tympanic membrane, the remnants of the hemostatic sponge were revealed (Figure $3 d$ ).

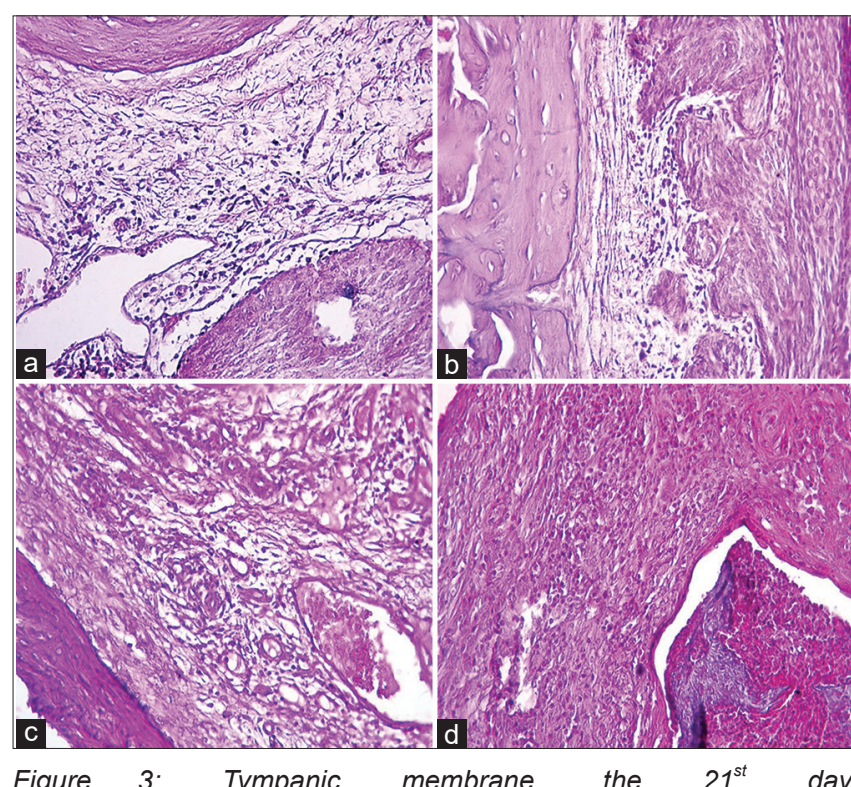

Figure 3: Tympanic membrane, the $21^{\text {st }}$ day (experiment, Group 01.1.1): Myringoplasty of the defect zone using preserved dura mater: (a-c) The formation of a delicate fibrous connective tissue with moderate cellular infiltration; (c) zone of vascularization by thin-walled vessels with diffuse lymphoid infiltration; (d) diffuse lymph-leukocyte infiltration in the border zone of the implant and the tympanic membrane tissue, the remnants of hemostatic sponge. Staining: Hematoxylin and eosin. Magnification: $\times 400$

After the end of the experiment, on the $30^{\text {th }}$ day after the plasty of the area of the tympanic membrane defect by preserved dura mater, the formation of coarse fibrous tissue with a chaotic arrangement of fibers was noted, diffuse infiltration with lymphocytes and individual foci of vascularization by capillary-type vessels remained in the edge zones of the implant (Figure 4), which indicated the continuing reparative process after injury.

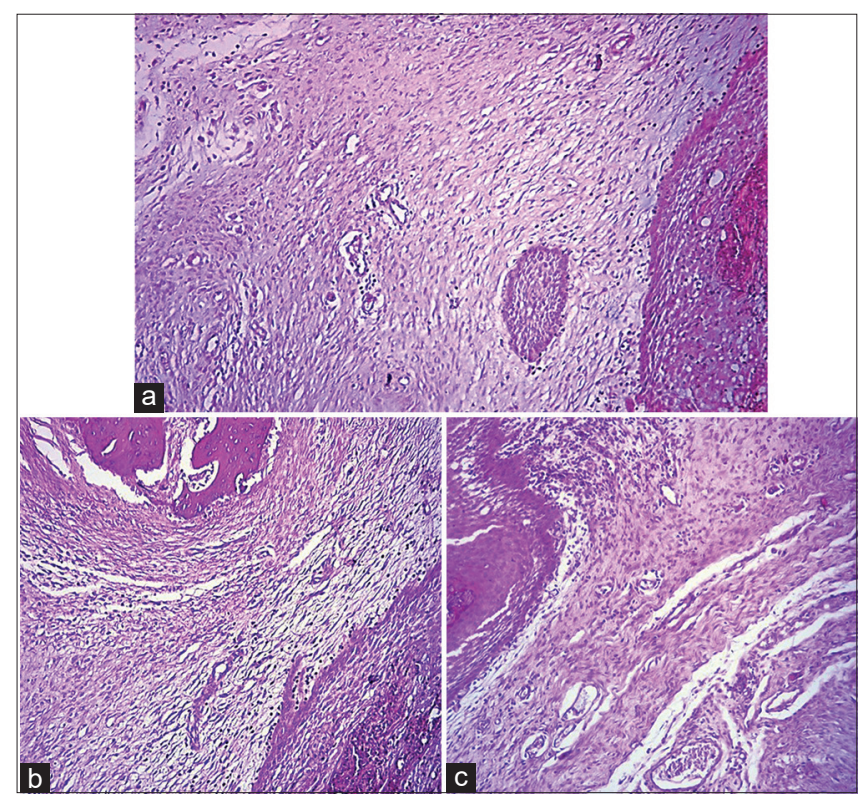

Figure 4: Tympanic membrane, the $30^{\text {th }}$ day - myringoplasty of the defect zone using preserved dura mater (experiment, Group 01.2.2): Coarse fibrous tissue formation, moderate lymphocyte infiltration in the marginal zone of the implant, separate foci of vascularization. Staining: Hematoxylin and eosin. Magnification: $a-\times 200 ; b, c-\times 400$ 
Inthe second group of the experiment, decellularized matrix of xenoperitoneum was used as an implant during myringoplasty to close the tympanic membrane defect, a morphological assessment of the dynamics of the reparative process was given at different times.

On the $7^{\text {th }}$ day of the experiment, after the implantation of the tympanic membrane defect zone with decellularized matrix of xenoperitoneum, the formation of young granulation tissue with the presence of tangles of small thin-walled capillary vessels in the structure of fibrous connective tissue was registered (Figure 5), cell infiltration did not reveal. The border zone with the tissues of the tympanic membrane in the form of hyaline cartilage and the remnants of the hemostatic sponge, used to stop bleeding, was well traced.

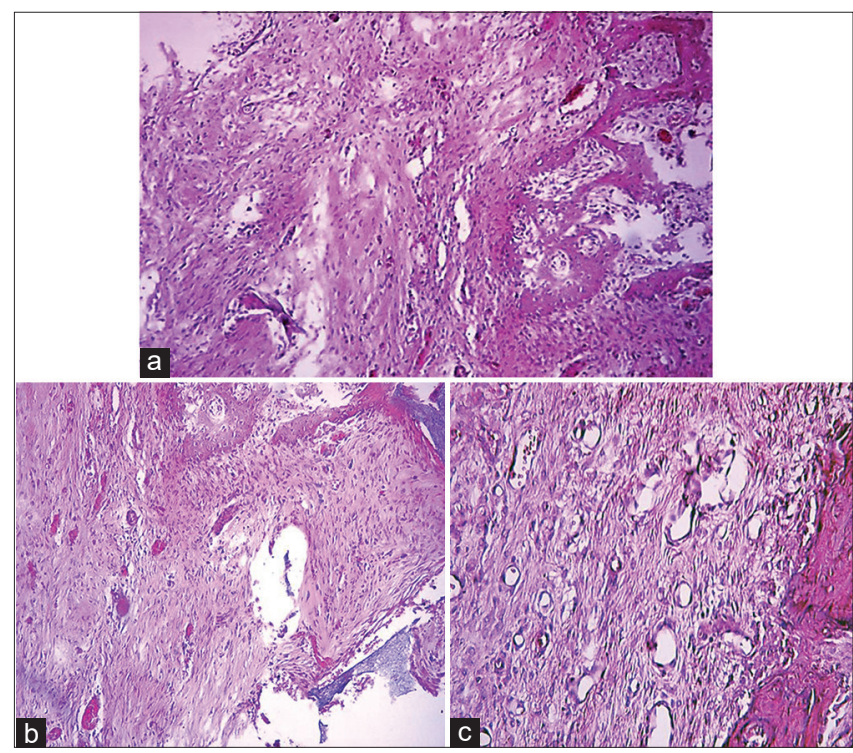

Figure 5: Tympanic membrane, implantation with decellularized matrix of xenoperitoneum, the 7th day: (a and b) - defect zone; (c) - interstitial edema, formation of granulatory tissue with tangles of small vessels. Staining: Hematoxylin and eosin. Magnification: $\times 200$

We also observed the formation of granulatory tissue after myringoplasty with decellularized matrix of xenoperitoneum in other groups at the same time. This process was represented by fibrous tissue with the presence of capillary vessels, moderate interstitial tissue edema was observed. Infiltration by macrophages and lymphocytes was not registered (Figure 6).

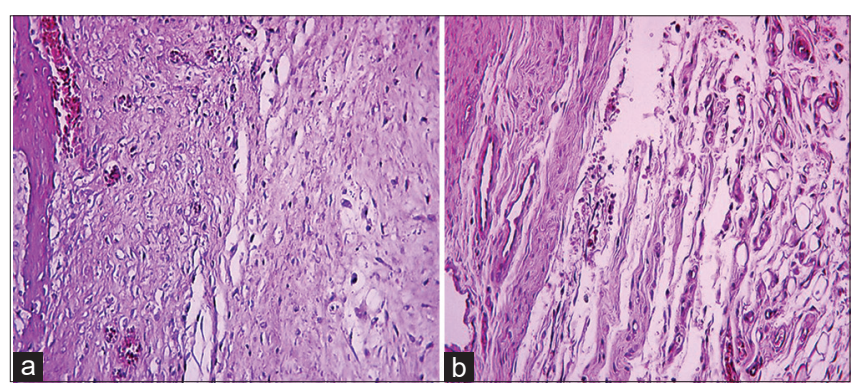

Figure 6: Tympanic membrane, myringoplasty with decellularized matrix of xenoperitoneum, the $7^{\text {th }}$ day: (a) The formation of granulation tissue in the implant area, the presence of capillary vessels in the structure of fibrous connective tissue; (b) interstitial edema. Staining: Hematoxylin and eosin. Magnification: $a-\times 100 ; b-\times 200$
Histological examination of the tympanic membrane implant zone on the $21^{\text {th }}$ day after myringoplasty with decellularized matrix of xenoperitoneum revealed the maturation of fibrous connective tissue, which was characterized by a bundle of fibers; remnants of a hemostatic sponge were detected in the boundary zone of the implant. There was also a decrease in the number of capillary vessels. We have already observed in other groups of the experiment at the same time that the bundles of fibers assumed a parallel arrangement during the formation of connective tissue (Figure 7).

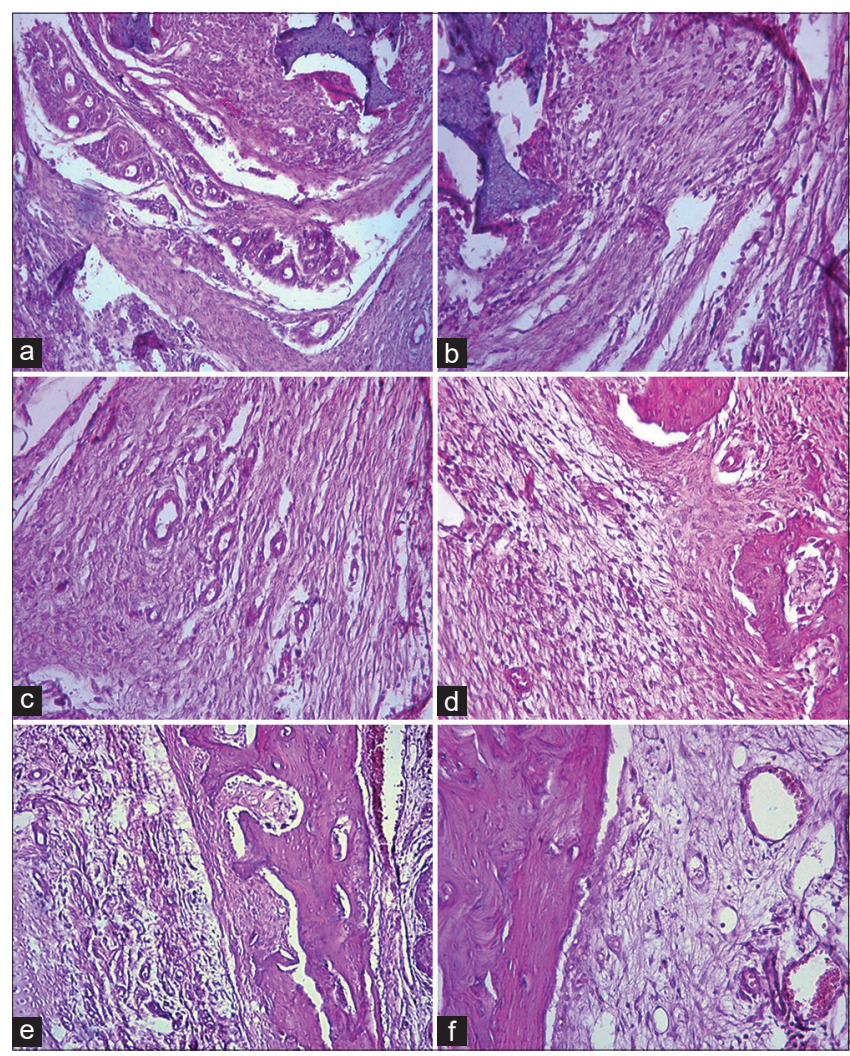

Figure 7: Tympanic membrane, myringoplasty with decellularized matrix of xenoperitoneum, period, the 21st day: (a) - Maturation of fibrous connective tissue with bundle arrangement of fibers, the remains of a hemostatic sponge in the border zone; ( $b$ and $c$ ) - A decrease of vessels number; ( $d$,e and f) -the formation of connective tissue with a parallel arrangement of fibers. Staining: Hematoxylin and eosin. Magnification: $a, b-\times 100: b, c-\times 200$

The formation of mature fibrous connective tissue with bundle and parallel arrangement of fibers between the elements of the cartilaginous tissue and the epidermis was registered in other groups during histological examination (Figure 8).

Thus, within, the dynamics of reparative processes after myringoplasty by decellularized matrix of xenoperitoneum with the formation of fibrous tissue was noted on the $21^{\text {nd }}$ day.

On the $30^{\text {th }}$ day of the experiment, histological examination of the tympanic membrane defect with the decellularized matrix of xenoperitoneum after myringoplasty showed that during this period of the experiment, the formation of mature fibrous connective 
tissue occurred, where there was the regression of capillary-type vessels, the cellular lymphoidmacrophage reaction was not revealed, which was a reflection of the positive dynamics of reparative process (Figure 8).

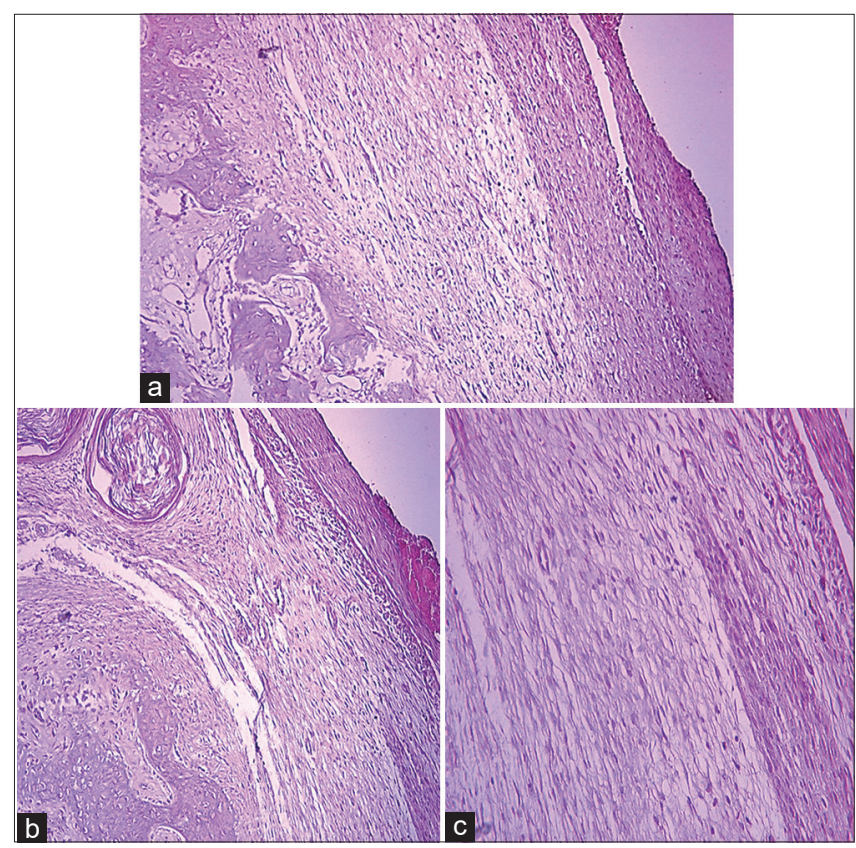

Figure 8: (a-c) Tympanic membrane, the $30^{\text {th }}$ day. Myringoplasty with decellularized matrix of xenoperitoneum: The formation of mature fibrous connective tissue with parallel arrangement of bundles of connective tissue fibers. Staining: Hematoxylin and eosin. Magnification: $a-\times 200 ; b, c-\times 400$

The formation of mature fibrous tissue was also noted in the border zone where fragments of the remains of the hemostatic sponge were detected in other groups of the experiment (Figure 9).

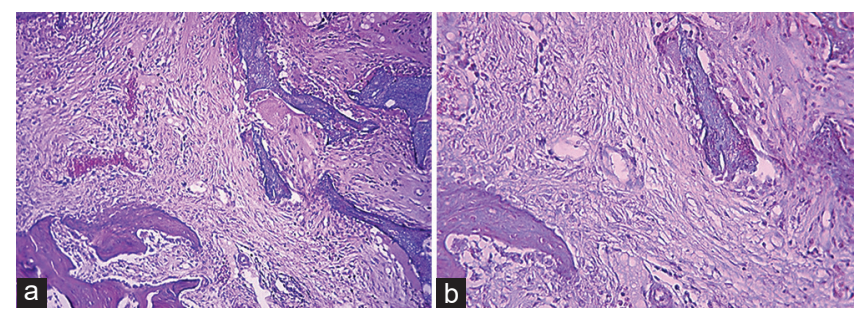

Figure 9: ( $a$ and b) Tympanic membrane, the $30^{\text {th }}$ day. Myringoplasty with decellularized matrix of xenoperitoneum: The formation of coarse fibrous tissue with a tendency to parallel bundle arrangement of connective tissue fibers in the border zone. Staining: Hematoxylin and eosin. Magnification: $\times 200$

\section{Discussion}

Analysis of the obtained data showed that the maturation of connective tissue in both groups was completed by the $30^{\text {th }}$ day. However, the wound process currency, the change of exudation, and proliferation phases in the experimental group and the comparison group differed in terms of timing.
Thus, the formation of young granulation tissue was recorded on the $7^{\text {th }}$ day in the experimental group with the use of decellularized matrix of xenoperitoneum, which characterizes the exudative phase of the tissue reaction in response to implantation and surgical trauma. The forming granulation tissue with newly formed thinwalled vessels of capillary type in the structure of the fibrous connective tissue, cellular infiltration was not registered.

An identical morphological picture was observed in the comparison group with a predominance of granulocytic cells in the cellular infiltrate. However, infiltration by macrophages and lymphocytes was observed in the area of implantation in the marginal zones, which indicated the transition of the exudative phase to the proliferative phase of the wound process.

The formation of mature fibrous connective tissue with bundle and parallel arrangement of fibers was registered in the experimental group after myringoplasty with decellularized matrix of xenoperitoneum on the $21^{\text {st }}$ day of observation. Thus, the dynamics of reparative processes after myringoplasty with the formation of fibrous tissue was noted; the development of a reactive or persistent inflammatory process in the forming scar was not revealed.

The morphological picture of the implantation zone was characterized by maturation and the formation of delicate fibrous connective tissue in the stroma with moderate infiltration by lymphocytes and macrophages by the $21^{\text {st }}$ day in the comparison group after myringoplasty with the use of preserved dura mater. The vascularization zone by thin-walled vessels with diffuse lymphoid infiltration was well traced. Granulocytic and lymphocytic cells with stromal cells were registered in the histological study of the material. This phenomenon can be regarded as a delay in the change of the exudative phase into the proliferative phase, which indicates the persistence of the reactive inflammatory process for up to the $21^{\text {st }}$ day.

The formation of mature fibrous connective tissue with regression of capillary vessels was observed in the experimental group after myringoplasty with decellularized matrix of xenoperitoneum on the $30^{\text {th }}$ day. There was no cellular lymphoid-macrophage reaction, which was a reflection of the positive dynamics of the reparative process.

The formation of coarse fibrous tissue with a chaotic arrangement of fibers was observed in the comparison group with the use of preserved dura mater by the $30^{\text {th }}$ day, diffuse infiltration with lymphocytes, and separate foci of vascularization by capillary-type vessels remained in the marginal zones of the implant, and it indicated the continuing reparative process after injury. 


\section{Conclusion}

Based on the foregoing, it can be noted that the experimental group using the decellularized matrix of xenoperitoneum had better reparative properties by the $30^{\text {th }}$ day. Furthermore, the change in the exudative phase into the proliferative phase occurred faster, and it indicated the absence of persistence of reactive inflammatory disease at an earlier date.

\section{References}

1. Abatov NT, Badyrov RM, Abatova AN, Asamidanov EM, Kaukenov BN. Biological implants in abdominal wall hernia repair (review). Georgian Med News. 2016;251:7-12. PMid:27001778

2. Qin $H$, Sun J, Li $X$, Liu $Y$, Jia Z. An experimental study on tympanic membrane reconstruction with acellular dermal matrix. Acta Otolaryngol. 2012;132(12):1266-70. https://doi.org/10.310 9/00016489.2012.701327

PMid:22831646

3. Ivanova NI, Dolgov VA, Shevlyuk NN, Fedyunin PS, Denner VA Efficiency of using nanostructured biological material in myringoplasty of acute post-traumatic tympanic membrane defects. Almanac Young Sci. 2016;4:27-8.

4. Kurmashova LM, Sopko ON, Bolozneva EV. Clinical results of myringoplasty in acute traumatic perforations. Russ Otorhinolaryngol. 2014;1:126.

5. Yawn RJ, Dedmon MM, O'Connell BP, Virgin FW, Rivas A Tympanic membrane perforation repair using porcine small intestinal submucosal grafting. Otol Neurotol. 2018;39(5):e332-5. https://doi.org/10.1097/mao.0000000000001792 PMid:29738388

6. Demirdelen S, Imamoğlu M. Arslan S. Topical dexpanthenol application improves healing of acute tympanic membrane perforations: An experimental study. ENT Updates. 2016;6(3):116-20. https://doi.org/10.2399/jmu.2016003007

7. Karataylı Özgürsoy S, Tunçkaşık ME, Tunçkaşık F, Akıncıoğlu E, Doğan $H$, Kocatürk $S$. Platelet-rich plasma application for acute tympanic membrane perforations. J Int Adv Otol. 2017;13(2):195-9. https://doi.org/10.5152/iao.2016.2533 